\section{Apigenin induces dermal collagen synthesis via smad2/3 signaling pathway}

\author{
Y. Zhang, ${ }^{1}$ J. Wang, ${ }^{1}$ X. Cheng, ${ }^{2}$ B. Yi, ${ }^{3}$ \\ X. Zhang, ${ }^{4}$ Q. Li ${ }^{1}$ \\ 'Department of Plastic and \\ Reconstructive Surgery, Shanghai $9^{\text {th }}$ \\ People's Hospital, School of Medicine, \\ Shanghai Jiao Tong University \\ 2Department of Urology, Renji Hospital, \\ School of Medicine, Shanghai Jiao Tong \\ University \\ ${ }^{3}$ Clinical College of General Hospital of \\ Beijing Military Region, Anhui Medical \\ University, Hefei \\ ${ }^{4}$ The Key Laboratory of Stem Cell \\ Biology, Institute of Health Sciences, \\ Shanghai Institutes for Biological \\ Sciences, Chinese Academy of Sciences \\ and Shanghai Jiao Tong University School \\ of Medicine, Shanghai, China
}

\section{Abstract}

Decrease in fibroblast-produced collagen has been proven to be the pivotal cause of skin aging, but there is no satisfactory drug which directly increases dermal thickness and collage density. Here we found that a flavonoid natural product, apigenin, could significantly increase collagen synthesis. NIH/3T3 and primary human dermal fibroblasts (HDFs) were incubated with various concentrations of apigenin, with dimethyl sulfoxide (DMS0) serving as the negative control. Real-time reversetranscription polymerase chain reaction (PCR), Western Blot, and Toluidine blue staining demonstrated that apigenin stimulated type-I and type-III collagen synthesis of fibroblasts on the mRNA and protein levels. Meanwhile, apigenin did not induce expression of alpha smooth muscle actin ( $\alpha$-SMA) in vitro and in vivo, a fibrotic marker in living tissues. Then the production of collagen was confirmed by Masson's trichrome stain, Picrosirius red stain and immunohistochemistry in mouse models. We also clarified that this compound induced collagen synthesis by activating smad2/3 signaling pathway. Taken together, without obvious influence on fibroblasts' apoptosis and viability, apigenin could promote the type-I and type-III collagen synthesis of dermal fibroblasts in vitro and in vivo, thus suggesting that apigenin may serve as a potential agent for esthetic and reconstructive skin rejuvenation.

\section{Introduction}

Dermis consists of several structural components, and collagen takes the major part. In addition to glycosaminoglycans and elastin fibers, dermal matrix in adult skin are composed of type I (80-85\%) and type III collagen $(10-15 \%) .{ }^{1}$ It is of great importance that collagen plays a main role in the texture and appearance of skin. Skin aging is inevitably associated with a disturbance in collagen metabolism $^{2}$ (due to the decreased activity of fibroblasts and their collagen synthesis), as well as elastin. Increasing the collagen content of dermis has been regarded as a well-effective way for anti-aging in skin.

Collagen is a kind of biomacromolecule and it cannot be absorbed through the stratum corneum. Recently, some researches demonstrated that collagen hydrolysate ingestion might be beneficial to slow chronological skin aging $^{3}$ and photoaging ${ }^{4}$ in rats, and the density of collagen fibrils increased compared with lactalbumin and water controls. ${ }^{5}$ But the clinical effect of oral collagen hydrolysate still lacks convincing evidences. Up to now, injectable collagen $^{1}$ or analogous composition ${ }^{6}$ filling implants are recognized as the well-accepted treatment modality for cosmetic purposes. However, maintaining skin appearance relies on expansive and complex treatment repeatedly because of the short-term duration of exogenic injected collagen.

Apigenin (4,5,7-trihydroxyflavone), a flavone subclass of flavonoid widely distributed in many herbs, fruits, and vegetables, is a substantial component of the human diet and has been shown to possess a variety of biological characteristics, including anti-oxidative, ${ }^{7}$ antiinflammatory effect, ${ }^{8}$ tumor growth inhibition ${ }^{9}$ and promoting neurogenesis. ${ }^{10}$ It has been shown that apigenin could enhance wound healing and tissue repair in diabetic rat skin. ${ }^{11}$ In the process of wound healing, fibroblasts secreted collagen and the formation of collagen-rich granulation tissue are vital pathophysiological mechanisms for wound closure. ${ }^{12}$

Given this, we wonder what effect would apigenin have on fibroblasts and whether apigenin could induce collagen synthesis in normal human dermal fibroblasts. Consequently, we examined its effects on collagen synthesis in normal human dermal fibroblasts in vitro and tested its function in the skin aging mouse model induced by D-Galactose. Furthermore, we investigated the potential mechanism involved in the positive effects of apigenin on collagen expression in fibroblasts.
Correspondence: Xiaoling Zhang and Qingfeng $\mathrm{Li}$, Department of Plastic and Reconstructive Surgery, Shanghai $9^{\text {th }}$ People's Hospital, Shanghai Jiao Tong University School of Medicine, 639 Zhizaoju Road, Shanghai 200011, China.

Tel. +86.21.23271699-5615 - Fax: +86.21.63089567. E-mails: (X. Zhang) xlzhang@sibs.ac.cn; (Q. Li) dr.liqingfeng@yahoo.com

Key words: Apigenin, flavonoid, collagen I/II, fibroblasts, smad2/3.

Contributions: YZ and JW contributed equally to this work; YZ, JW, experimental work, data collection and interpretation; $\mathrm{XC}$, participation in experimental work design and coordination, data acquisition; BY, participation in study design, data collection, analysis of data and manuscript preparation; QL, XZ, study design, data analysis and interpretation, manuscript drafting.

Conflict of interest: the authors declare no conflict of interest.

Funding: this study was supported by grants from the key project of the National Natural Science Foundation (No. 81230042), the National Key Project of Scientific and Technical Supporting Programs Funded by Ministry of Science \& Technology of China (No. 2012BAI11B03) (Q. Li) and the Chinese Academy of Sciences (No. XDA01030102), Shanghai Municipal Commission of Health and Family Planning (No. 2013ZYJB0501) (X. Zhang).

Received for publication: 28 November 2014 . Accepted for publication: 9 March 2015.

This work is licensed under a Creative Commons Attribution NonCommercial 3.0 License (CC BYNC 3.0).

(C) Copyright Y. Zhang et al., 2015 Licensee PAGEPress, Italy

European Journal of Histochemistry 2015; 59:2467 doi:10.4081/ejh.2015.2467

\section{Materials and Methods}

\section{Cell culture}

Primary human dermal fibroblasts were obtained from adolescent foreskin tissue of ten people (aged 8-12 years), and none of them had a history with skin diseases. Skin tissue was obtained after obtaining informed consent from the patients, with the approval of the ethics committee of Shanghai $9^{\text {th }}$ People's Hospital and in conformity with the Helsinki guidelines. NIH/3T3 and human dermal fibroblasts (HDFs) were maintained in DMEM (Hyclone, Thermo Fisher Scientific, Waltham, MA, USA) supplemented with $10 \%$ fetal bovine 
serum (Hyclone, Thermo Fisher Scientific), $100 \mathrm{U} / \mathrm{mL}$ penicillin, and $100 \mathrm{mg} / \mathrm{L}$ streptomycin. Both HDFs and NIH/3T3 were incubated at $37^{\circ} \mathrm{C}$ in a humidified atmosphere with $5 \%$ $\mathrm{CO}_{2}$. Primary fibroblasts of passages $6-8$ were used. Toluidine blue (Sigma-Aldrich, St. Louis, M0, USA) staining was used to assess extracellular matrix synthesis. ${ }^{13}$

\section{Real-time PCR analysis}

The total RNA of cells was isolated using TRIzol reagent (Invitrogen, Carlsbad, CA, USA) and subjected to reverse transcription with Oligo (dT) and M-MLV Reverse Transcriptase (Thermo Fisher Scientific). Synthesized complementary DNA (cDNA) was analysed with quantitative real-time PCR using SYBR® Premix (Takara, Dalian, China) and Roche480 system. Glyceraldehyde 3-phospate dehydrogenase (GAPDH) was used as a reference gene. Primers sequences were as follows: collagen, type I, alpha 2 (Colla2; mouse), 5 -GGTGAGCCTGGTCAAACGG-3 (forward) and 5 - ACTGTGTCCTTTCACGCCTTT-3 (reverse); Colla2 (human), 5 -GGCCCTCAAGGTTTCCAAGG-3 (forward) and 5-CACCCTGTGGTCCAACAACTC-3 (reverse); collagen, type III, alpha 1 (Col3al; mouse), 5 - CTGTAACATGGAAACTGGGGAAA-3 (forward) and 5 CCATAGCTGAACTGAAAACCACC-3 (reverse); Col3al (human), 5 -TTGAAGGAGGATGTTCCCATCT-3 (forward) and 5- ACAGACACATATTTGGCATGGTT-3 (reverse); matrix metalloproteinases 1 (MMP1; human), 5 AAAATTACACGCCAGATTTGCC-3 (forward) and 5 -GGTGTGACATTACTCCAGAGTTG-3 (reverse); matrix metalloproteinases 2 (MMP2; human), 5 -TACAGGATCATTGGCTACACACC-3 (forward) and 5-GGTCACATCGCTCCAGACT-3 (reverse); matrix metalloproteinases 9 (MMP9; human), 5 -TGTACCGCTATGGTTACACTCG-3 (forward) and 5 GGCAGGGACAGTTGCTTCT-3 (reverse); tissue inhibitor of metalloproteinases1 (TIMP1; human), 5 -CTTCTGCAATTCCGACCTCGT-3 (forward) and 5-ACGCTGGTATAAGGTGGTCTG-3 (reverse); $\alpha$-SMA (human), 5 AAAAGACAGCTACGTGGGTGA-3 (forward) and 5 -GCCATGTTCTATCGGGTACTTC-3 (reverse).

\section{Cell viability assay}

For the cell viability assay, HDFs were seeded on 96-well plates (100 mL per well), followed by apigenin (cat. no. 42251; SigmaAldrich) or DMSO (Sigma-Aldrich) treatment. After 3 or 5 days, cell culture medium was replaced by Thiazolyl Blue Tetrazolium Bromide (MTT) working solution, followed by a 4-hour incubation at $37^{\circ} \mathrm{C}$ in a $5 \% \mathrm{CO}_{2}$ incubator. After MTT working solution was removed and DMSO added, the absorbances at $490 \mathrm{~nm}$ were detected.

\section{Flow cytometric analysis}

Cell apoptosis was assessed by flow cytometry using the Alexa Fluor ${ }^{\circledR} 488$ Annexin V/Dead Cell Apoptosis Kit (Invitrogen, Carlsbad, CA, USA). Following apigenin (5 $\mu \mathrm{mol} / \mathrm{L}$ or 1 $\mu \mathrm{mol} / \mathrm{L}$ ) or dimethyl sulfoxide (DMSO) treatment, harvested cells were suspended in 100 $\mu \mathrm{L}$ Annexin-binding buffer. Then, $5 \mu \mathrm{L}$ Alexa Fluor® 488 Annexin V and $1 \mu \mathrm{L}$ PI working solution were added and incubated with the cells for 15 min in the dark. After the incubation period, $400 \mu \mathrm{L} 1 \mathrm{X}$ Annexin-binding buffer was added and mixed gently. The stained cells were analysed directly by flow cytometry using the Cell Quest program (Becton Dickinson, CA, USA). Data were analysed using FlowJo software.

\section{Colony formation assay}

Anchorage-dependent growth of HDFs were investigated by monolayer colony formation assay. ${ }^{14}$ Cells were cultured in a 6 -well plate (500 per well) and treated with $5 \mu \mathrm{mol} / \mathrm{L}$ or 1 $\mu \mathrm{mol} / \mathrm{L}$ apigenin or DMSO. After cultured for 14 days, surviving colonies were stained $5 \mathrm{~min}$ with Gentian Violet (Sigma-Aldrich) after 4\% paraformaldehyde fixation.

\section{Immunofluorescence cell staining}

Human dermal fibroblasts at a density of $2 \times 10^{3}$ cells per well were seeded on cover slides in 24-well plates and incubated overnight. Cells were fixed with $4 \%$ paraformaldehyde and blocked with $5 \%$ goat serum in PBST $(0.1 \%$ TritonX-100 in phosphate buffered saline) for $1 \mathrm{~h}$. For F-actin staining, cells were incubated with Alexa Fluor 488 Phalloidin (Cytoskeleton Inc., Denver, C0, USA; $1: 200$ ) for $1 \mathrm{~h}$ at room temperature. For $\alpha$-SMA staining, cells were incubated with primary antibodies against $\alpha$-SMA (Abcam, Cambridge, UK, 1:200) for $2 \mathrm{~h}$ at room temperature, followed by an Alexa Fluor 555-conjugated secondary antibody. For smad3 staining, cells were incubated with primary antibodies against smad3 (Cell Signaling Technology, Beverly, MA, USA; 1:200) for $2 \mathrm{~h}$ at room temperature, followed by an Alexa Fluor 488-conjugated secondary antibody. Immunofluorescence signals were captured using confocal microscopy (LSM 510, META Laser Scanning Microscope; Zeiss, Jena, Germany).

\section{Smad2/3 knockdown by siRNA}

RNA interference was performed using smad2/3 siRNA (human) (sc-37238; Santa Cruz Biotechnology, Dallas, TX, USA), targeting human smad2/3 and control siRNA (sc37007) as negative control. Transfection for HDFs was conducted using Lipofectamine RNAiMAX reagent (Invitrogen, Carlsbad, CA, USA) according to the manufacturer's protocol.

\section{Western blot}

Cultured cells were lysed using radioimmunoprecipitation assay (RIPA) lysis buffer. Protein concentrations were determined using a micro bicinchoninic acid (BCA) assay (Thermo Fisher Scientific). Twenty micrograms total protein extract was separated by $8 \%$ or $10 \%$ sodium dodecyl sulfate-polyacrylamide gel electrophoresis (SDS-PAGE) under reducing conditions and electroblotted onto polyvinylidene difluoride membranes (Millipore, Bedford, MA, USA). The membrane was then blocked and then incubated with primary antibodies overnight at $4^{\circ} \mathrm{C}$.

The primary antibodies used included the followings: anti-Col1a2, anti-Col3a1, anti- $\alpha$ SMA (Abcam; 1:1000), anti-cyclin-dependent kinase (CDK) family, anti-cyclin D1, anticyclin E1, anti-smads, anti-MAPK family (Cell Signaling Technology, Beverly, MA, 1:1000), anti-GAPDH (Sigma-Aldrich; 1:10,000). Immunoreactive bands were quantitatively analyzed with ImageJ software.

\section{D-galactose-induced skin aging mouse model and animal \\ experiments}

Six-week-old female C57BL/6 mice were purchased from the Shanghai Slac Laboratory Animal (Slac, Shanghai, China). All animal studies have been approved by the Animal Care and Use Committee of Shanghai Jiao Tong University. All efforts were made to minimize animal suffering.

A total of 18 mice were randomly assigned to three groups $(n=6)$. Two groups of animals received daily subcutaneous injection of Dgalactose (D-gal; $1000 \mathrm{mg} / \mathrm{kg}$ ) for 8 weeks. ${ }^{15}$ The third group received phosphate buffered saline (PBS) as a negative control. Two weeks later, DMSO and apigenin $(5 \mu \mathrm{mol} / \mathrm{L})$ was delivered by microneedles ${ }^{16}$ [MTS-Roller Model: CR2 $(0.2 \mathrm{~mm})]$ to the dermis of Dgalactose treated mice once a day for 4 weeks, respectively. Mice were sacrificed at the end of treatment, and skin tissue was harvested for further analyses.

\section{Histology and immunohistochemistry}

Paraformaldehyde-fixed paraffin-embedded tissue sections $(5 \mu \mathrm{m})$ were stained with hematoxylin and eosin (H\&E), Masson's trichrome (Trichrome stain LG solution, HT10316; Sigma-Aldrich) and Picrosirius red (Fluka, Buchs, Switzerland). For immunohistochemical staining, the sections were detected with primary antibodies against collagen I/II (Millipore; 1:1,000) and $\alpha$-SMA (Abcam; 1:200) overnight at $4^{\circ} \mathrm{C}$. After incubation with the appropriate secondary antibodies, the sections were developed with diaminobenzidine and counterstained with hematoxylin. 


\section{Statistical analysis}

Statistical differences were calculated using Friedman's analysis of variance (ANOVA), with post-hoc least significant difference (LSD) test as appropriate. A significant difference among groups was set at $\mathrm{P}<0.05$.

\section{Results}

Apigenin stimulated collagen synthesis but had no effect on matrix metalloproteinases in vitro

Fibroblasts are the predominant mesenchy- mal cells in the dermis, and their function is strongly implicated in dermatology. To study apigenin's effect on fibroblasts (Figure 1A), NIH/3T3 and HDFs were administered with apigenin at the concentrations of $5 \mu \mathrm{mol} / \mathrm{L}$. As shown in Figure 1B, apigenin could potently increase Toluidine blue staining after apigenin treatment for 5 days, which mean the
A<smiles>O=c1cc(-c2ccc(O)cc2)oc2cc(O)cc(O)c12</smiles>

B

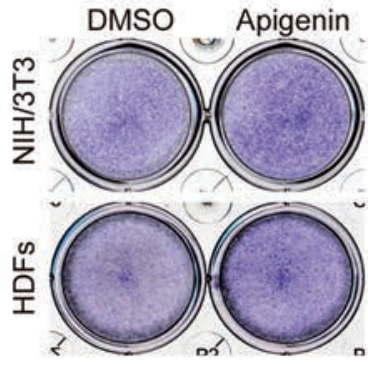

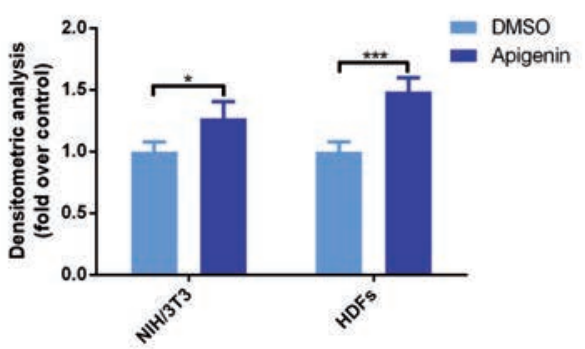

C
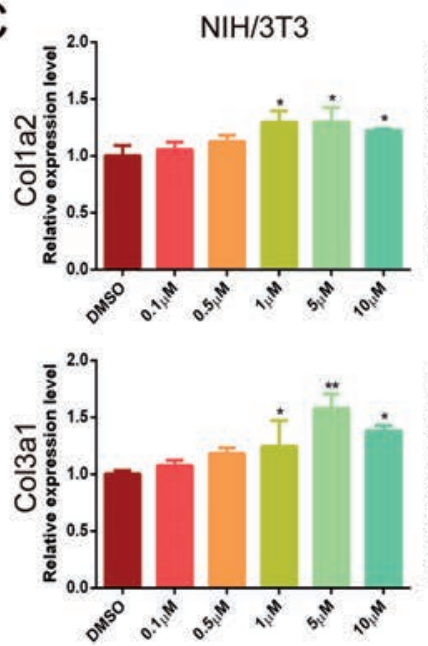

E
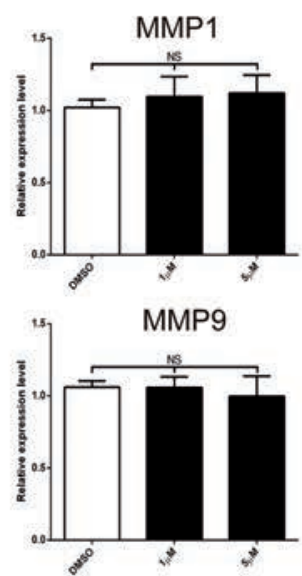
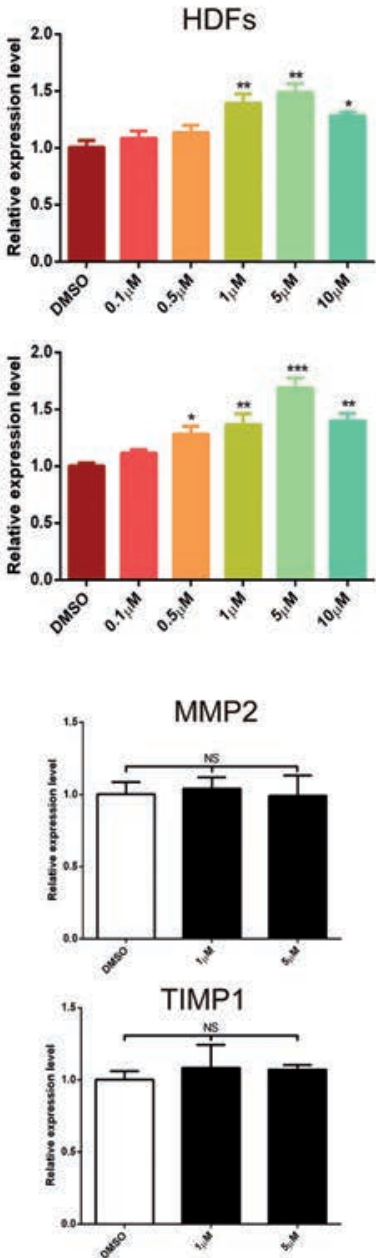

D
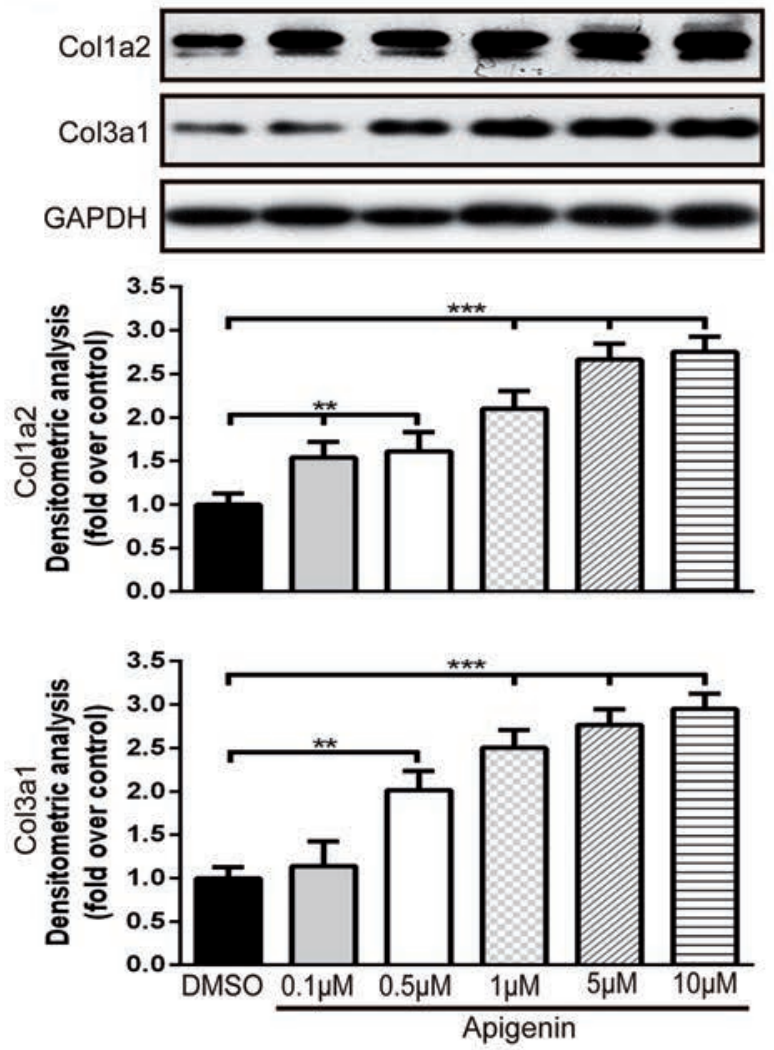

Figure 1. Apigenin stimulated collagen synthesis of fibroblasts. A) The molecular structure of apigenin. B) Toluidine blue staining in NIH/3T3 and HDFs for 5 days. C) Dose-dependent effects of apigenin on mRNA expression of Col1a2 and Col3a1 in NIH/3T3 and HDFs for 3 days. D) The protein level of Col1a2 and Col3a1 was measured by Western Blot at 5 days after apigenin was applied at concentrations of $0.1 \mu \mathrm{mol} / \mathrm{L}$ to $10 \mu \mathrm{mol} / \mathrm{L}$. E) The expression of MMP1, MMP2, MMP9 and TIMP1 were also assessed by real-time PCR. Data are presented as mean $\pm S D, n \geq 3$; NS, not significant; ${ }^{*} \mathbf{P}<0.05 ;{ }^{* *} \mathbf{P}<0.01 ;{ }^{* * *} \mathbf{P}<0.001$. 
synthesis of extracellular matrix was increased. ${ }^{13}$ Further real-time PCR analysis showed that, in NIH/3T3 and HDFs, apigenin $(0.1 \mu \mathrm{mol} / \mathrm{L}-10 \mu \mathrm{mol} / \mathrm{L})$ dose-dependently stimulated endogenous expression of Colla2 and Col3al. The most significant changes were observed when NIH/3T3 and HDFs were treated with apigenin at the concentration of 5 $\mu \mathrm{mol} / \mathrm{L}$, and the increase of Col3al was more obvious than Colla2 (Figure 1C). These upregulation effect of apigenin on collagen expression were then confirmed by Western blot analysis. When HDFs were treated with apigenin for 5 days, the protein level of Colla2 and Col3al were higher than that of cells treated with DMSO (Figure 1D). In addition, we next examined the effect of apigenin on matrix metalloproteinases (MMPs) and
A

B

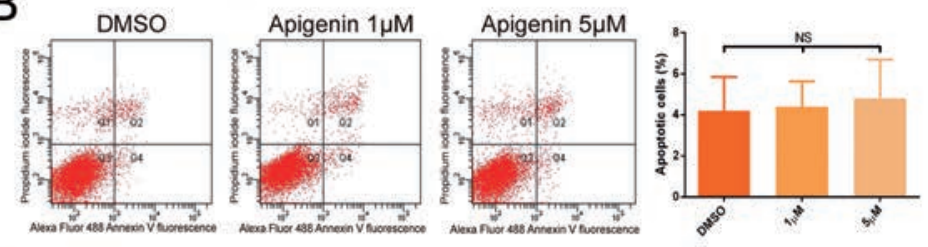

C
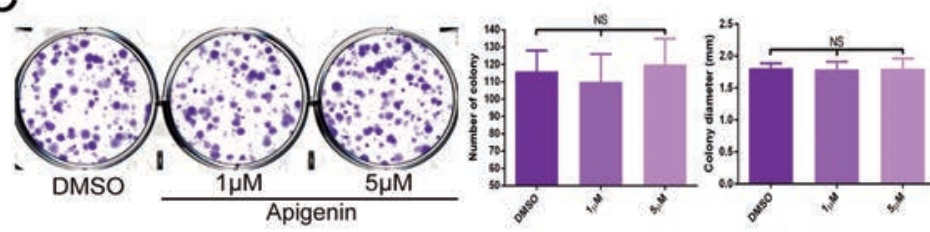

D
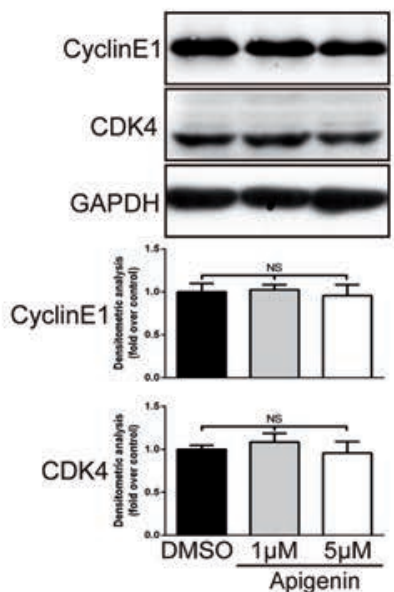

E

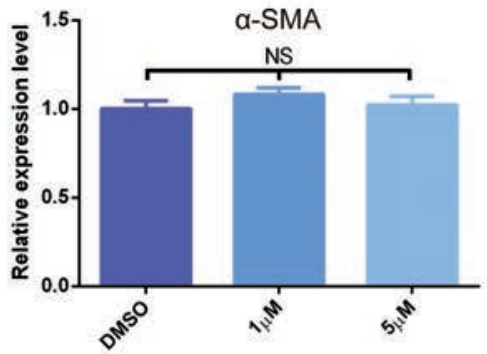

F

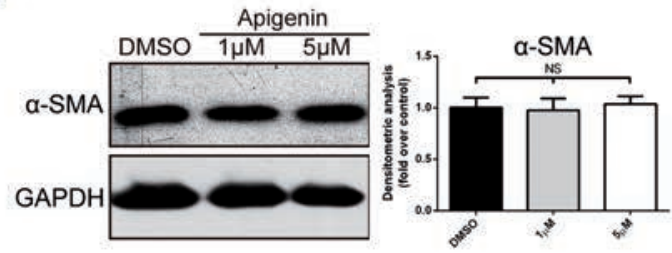

G

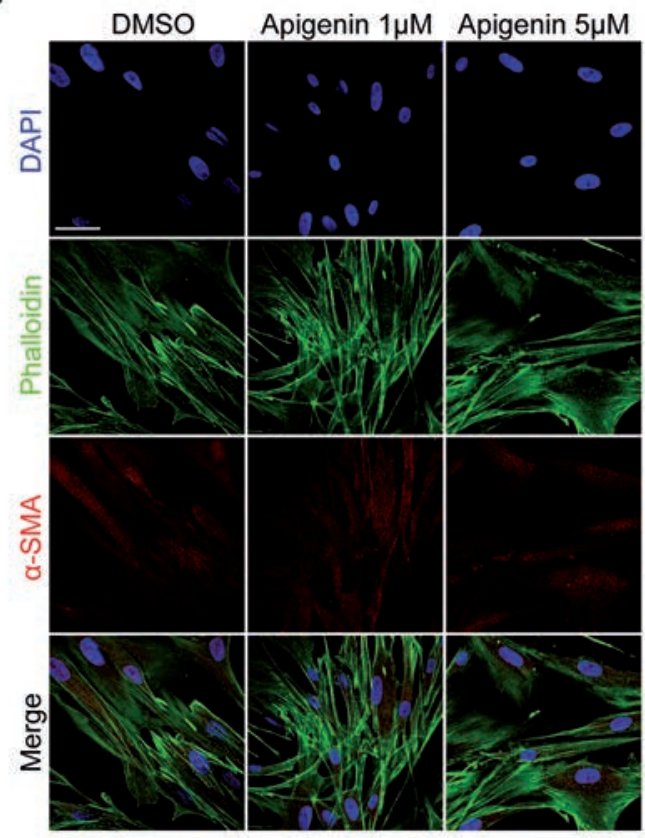

Figure 2. No obvious cytotoxicity exerted by apigenin on fibroblasts viability, apoptosis, proliferation, cell cycle and activation. A) Cell viability was examined by MTT assays at 3 or 5 days after apigenin was applied in HDFs. B) Apoptosis was evaluated after treating HDFs with $5 \mu \mathrm{mol} / \mathrm{L}$ or $1 \mu \mathrm{mol} / \mathrm{L}$ apigenin or DMSO; flow cytometry profile represents Alexa Fluor ${ }^{\circledR} 488$ Annexin V staining in X axis and PI in $\mathrm{Y}$ axis. C) The effect of apigenin on fibroblasts growth was investigated by monolayer colony formation assay. D) The expression of cyclin E1, CDK4, cyclin D1, CDK2 and p-CDK2 proteins was analysed using Western blot in HDFs. E-F) The levels of $\alpha$-SMA mRNA and protein expression were measured by real-time PCR and Western blot. G) Immunofluorescence cell staining for $\alpha$ SMA and F-actin in cultured HDFs after incubation with apigenin or DMSO for $72 \mathrm{~h}$; F-actin is shown by green fluorescence and $\alpha$ SMA is shown by red fluorescence; nucleus (blue) was stained with DAPI; scale bar: $50 \mu \mathrm{m}$. Data are presented as mean \pm SD, $\geq 3$; NS, not significant; ${ }^{* *} \mathbf{P}<0.01 ;{ }^{* *} \mathbf{P}<0.001$. 
TIMP1, well-known proteases that degrade collagen proteins. The expression of MMP1, MMP2, MMP9, and their inhibitor TIMP1 were unchanged (Figure 1E).

\section{Apigenin did not effect fibroblasts viability and activity}

Afterwards, we studied the effect of apigenin on fibroblasts viability, apoptosis, proliferation and activation. In vitro, MTT assays showed that the viability of HDFs was similar with those incubated with DMSO, when incubated with apigenin ( 1 to $10 \mu \mathrm{mol} / \mathrm{L})$ for 3 or 5 days, respectively (Figure 2A). In addition, no significant differences in percentages of apoptotic cells was observed after the exposure of fibroblasts to apigenin (Figure 2B). To investigate the effects of apigenin on proliferation and cell cycle, colony formation assay and Western Blot analysis of cell cycle related proteins were performed. Colony forming ability of HDFs was similar in apigenin-treated group to DMSO-treated group (Figure 2C). The expression levels of cell cycle associated proteins remained unchanged between apigenin and DMSO treated groups (Figure 2D). These results suggested that apigenin had no obvious cytotoxicity on fibroblasts' viability, apoptosis and proliferation.

Fibroblast overactivation leads to pathological collagen deposition or scar formation. ${ }^{17}$ Myofibroblasts, known as activated fibroblasts, are marked by $\alpha$-SMA expression. To determine the effects of apigenin on fibroblasts' activation, we evaluated the levels of $\alpha$-SMA mRNA and protein expression (Figure 2 E,F) in cultured HDFs treated with apigenin. We found that $\alpha$-SMA mRNA expression had no obvious change in apigenin-treated cells compared with DMSO-treated cells. We also showed that apigenin did not affect $\alpha$-SMA expression in vitro by immunofluorescence staining (Figure 2G). These findings suggested that apigenin did not cause fibroblasts to overactivate into myofibroblasts while collagen synthesis was increasing.

\section{Induction of collagen synthesis was mediated by smad $2 / 3$ activation}

To further explore the underlying mechanism of how apigenin activated type-I and type-III collagen gene expression, transforming growth factor beta 1 (TGF- $\beta 1$ ) and mitogen-activated protein kinase (MAPK) signaling pathway were analysed. TGF- $\beta 1$ is a prototypic fibrogenic cytokine, enhancing extracellular matrix gene expression. Previous studies proved that Colla2 and Col3al were direct TGF- $\beta 1 /$ smad3 targets in human dermal fibroblasts. ${ }^{18}$ As shown in Figure 3A, when HDFs were treated for $12 \mathrm{~h}$, apigenin (1 $\mu \mathrm{mol} / \mathrm{L}$ or $5 \mu \mathrm{mol} / \mathrm{L}$ ) markedly increased the expression of phosphorylated smad2 and smad3 in a dose-dependent manner, whereas total smad2, smad3 and smad4 did not obviously alter. It also showed that apigenin had sustained effect on promoting phosphorylation of smad2 and smad3 after a 3-day treatment (Figure 3B). Yoon et al..$^{19}$ revealed that MAPK pathway was involved with peptide-induced collagen synthesis of fibroblasts. However, when fibroblasts were treated with apigenin for $12 \mathrm{~h}$, the expression of total and phosphorylated JNK, ERK and p38 protein remained unchanged, compared with DMSO (Figure 3C). Immunofluorescence experiments demonstrated that after treatment with apigenin for $12 \mathrm{~h}$, smad3 protein (labeled by green) was significantly increased and mostly translocated into the nucleus (labeled by blue) (Figure 3D). By contrast, in the DMSO groups, smad3 were retained in the cytoplasm. Once targeted knockdown smad2/3 by specific siRNA, the upregulation effect of apigenin on the expression of collagen type-I and type-III protein was obviously reduced (Figure 3E), which confirmed that smad2/3 is required for the transduction of apigenin effect on collagen expressions.

\section{Apigenin stimulated collagen syn- thesis in the D-galactose-induced skin aging mouse model}

The in vivo effects of apigenin on collagen synthesis was investigated in the D-galactoseinduced skin aging mouse model. The collagen expression was showed by H\&E, Masson's trichrome stain, Picrosirius red stain and immunohistochemistry. Histology showed significant changes in dermal thickness and density in samples obtained from D-gal-treated mice compared with PBS control group (Figure 4 A-D). After 1 month of apigenin administration at the concentration of 5 $\mu \mathrm{mol} / \mathrm{L}$, the mice exhibited obviously increased dermal thickness and collagen density compared with DMSO-treated mice (Figure 4 A-D). Magnified images showed that dermis in the apigenin-treated group exhibited compact and clearly evident staining, whereas collagens were loosely distributed in DMSO-treated dermis of the aging skin model (Figure 4 A-D), in both Masson's trichrome stain, Picrosirius red stain and immunohistochemistry examinations. Dermal collagen could be subdivided into type I and type III collagen after Picrosirius red staining under polarized light. As shown in Figure 4F, apigenin could significantly increase both collagen type I and type III density in the dermis of the skin aging mouse model. Quantitative data of dermal thickness and collagen density showed that mice subcutaneously injected with D-gal showed thinner skin and less collagen compared to control mice and apigenintreated mice demonstrated significantly thick- er and compact dermis (Figure $4 \mathrm{E}, \mathrm{F}$ ). The in vivo study also demonstrates that apigenin does not show any effect on activation of fibroblasts (Figure 4G).

\section{Discussion}

Dermal atrophy is the major causes of aging appearance. ${ }^{20}$ In vivo and in vitro studies show that decline in the production of collagen in aging fibroblasts is mainly responsible for decreasing in dermal thickness seen in extrinsically aging skin, which reveals dermal atrophy, fragmentation, and irregular collagen bundles..$^{21}$ Since the 70 s, animal and human derived collagens have been studied for soft tissue augmentation. ${ }^{22}$ Injectable filling implants are now widely used for cosmetic purposes. However, exogenic injectable collagen often presented various complications such as allergy, ecchymosis, local necrosis and infections of bacteria or virus. Scientists have tried for decades to find other alternative to stimulate endogenous collagen synthesis. There are several anti-oxidants, such as vitamins $\mathrm{C}$ and $E$, co-enzyme $Q_{10}$ and retinoids used for treating UV-induced skin aging and wrinkles. ${ }^{23,24}$ However, only few compounds are able to induce type I collagen synthesis ${ }^{25-27}$ and none of them can stimulate both type I and type III collagen synthesis according to the record in literatures.

Apigenin, a plant flavone, has gained considerable attention due to its health benefits, chemopreventive properties and wide distribution in the plant kingdom..$^{28}$ Many studies have demonstrated that apigenin possesses a wide range of biological activities to the skin. It has been reported that apigenin can stimulate nucleotide excision repair genes to protect skin keratinocytes ${ }^{29}$ against UVB-induced skin inflammation. ${ }^{30}$ Dietary apigenin attenuates the development of atopic dermatitis-like skin lesions in atopic dermatitis model..$^{31}$ Apigenin could also effectively reduce the incidence and size of skin tumors caused by ultraviolet B (UVB) exposure through the enhancement of UVB-induced apoptosis. $^{32}$ In this study, we investigated the effect of apigenin on dermal fibroblasts' function.

At first, we found apigenin could increase the mRNA expression of Colla2 and Col3al in NIH/3T3 and HDFs. With extracellular matrix staining and Western Blot analysis, the stimulative effect of collagen on protein level was more significant. Although as reported in the literature, basal levels of Collal and $\alpha$-SMA mRNAs were reduced in fibroblasts treated with high concentration of apigenin (20 $\mu \mathrm{mol} / \mathrm{L}),{ }^{33}$ our research confirmed that apigenin of $25 \mu \mathrm{mol} / \mathrm{L}$ showed obvious cytotoxici- 
A

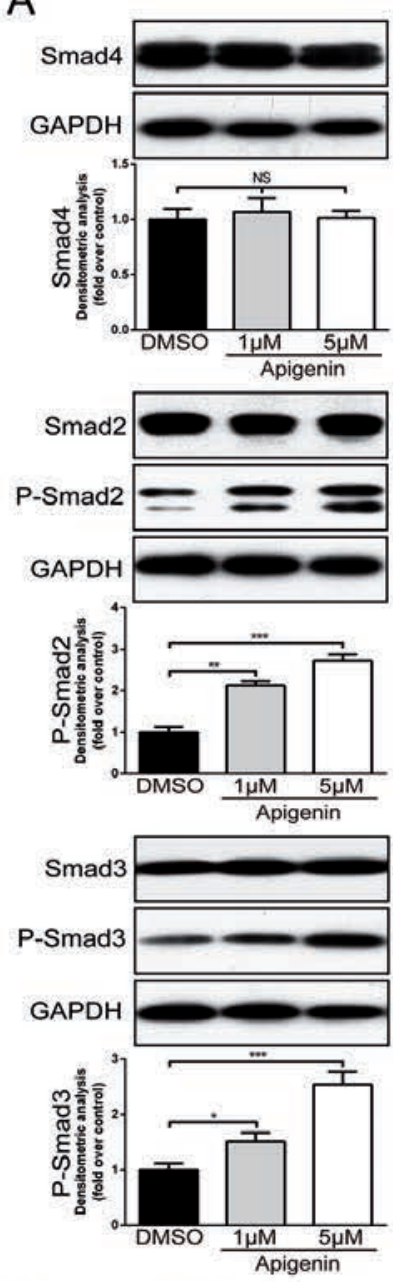

B
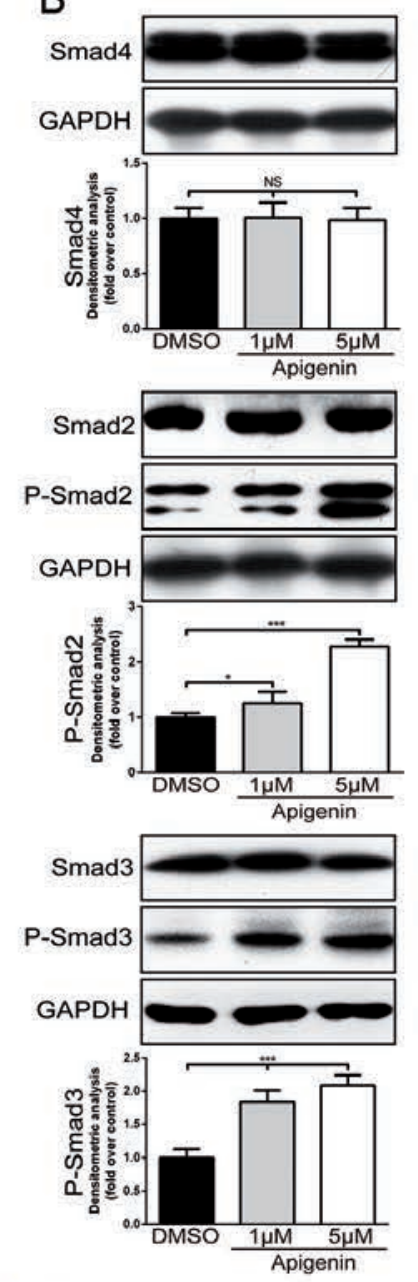

D

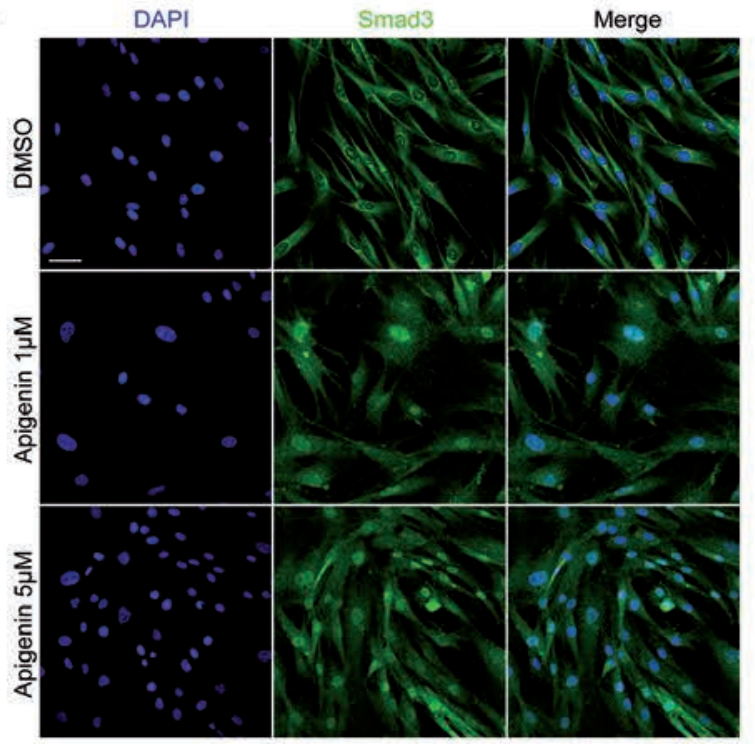

C

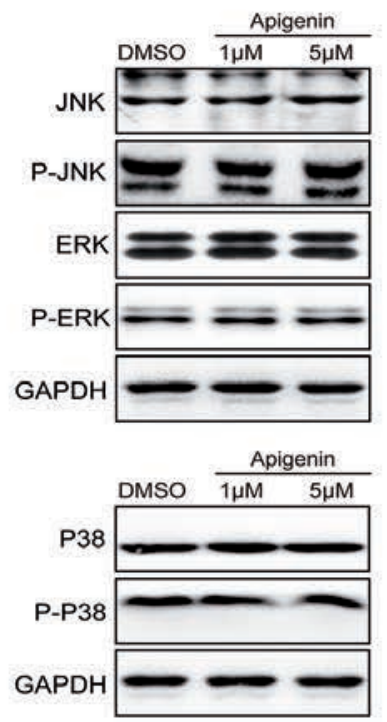

E
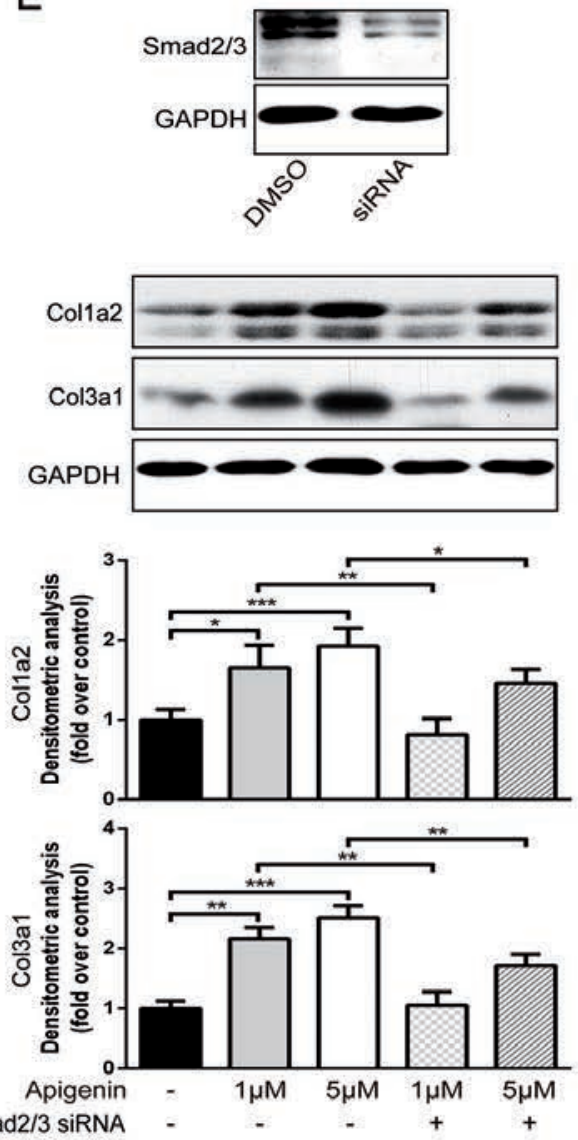

Figure 3. Apigenin-mediated collagen synthesis increase via smad2/3 signaling pathway. A,B) Western blot analysis and quantification of phosphorylated and total smad2, smad3 and total smad4 in HDFs with or without apigenin stimulation for $12 \mathrm{~h}$ or 3 days. C) Western Blot analysis of JNK, ERK and p38 in HDFs with or without apigenin stimulation for $12 \mathrm{~h}$. D) Immunofluorescence experiments: smad3 was labeled as green; nucleus (blue) was stained with DAPI; scale bar: $50 \mu \mathrm{m}$. E) Western blot showed the expression of Col1a2 and Col3a1 in HDFs when cells were treated with DMSO, apigenin or apigenin with specific siRNA of smad2/3. Data are presented as mean $\pm \mathrm{SD}, \mathrm{n} \geq 3$; NS, not significant; ${ }^{*} \mathrm{P}<0.05$; ${ }^{* *} \mathrm{P}<0.01$; ${ }^{* *} \mathrm{P}<0.001$. 
A

B
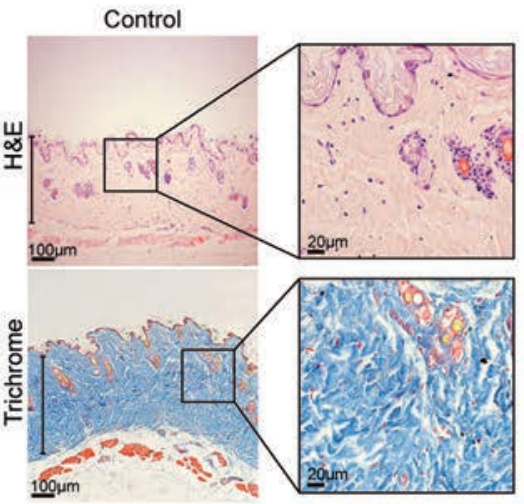

C

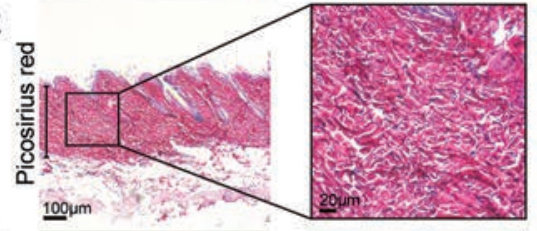

D

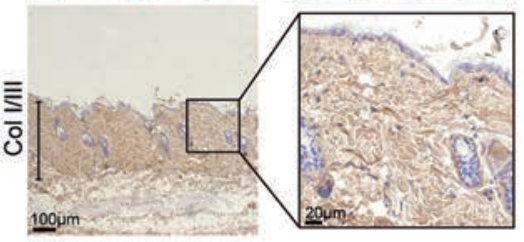

DMSO+D-gal
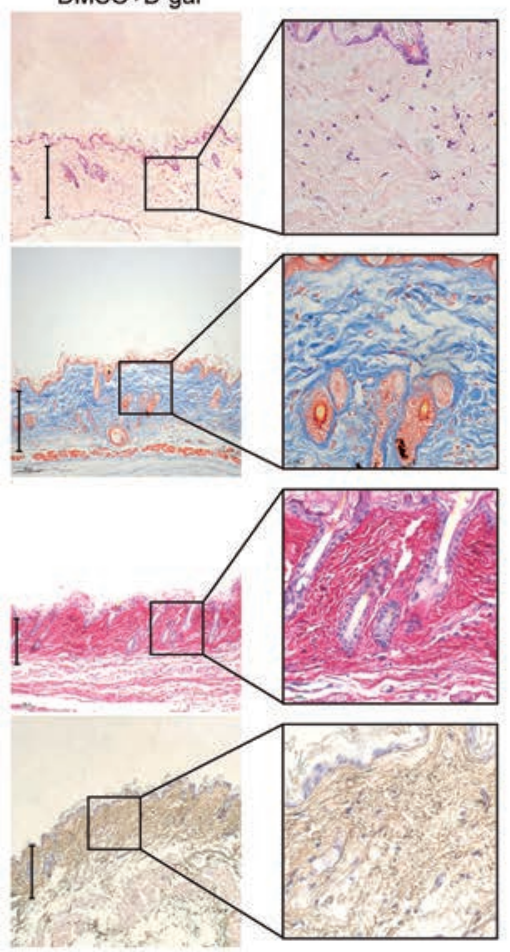

Apigenin+D-gal
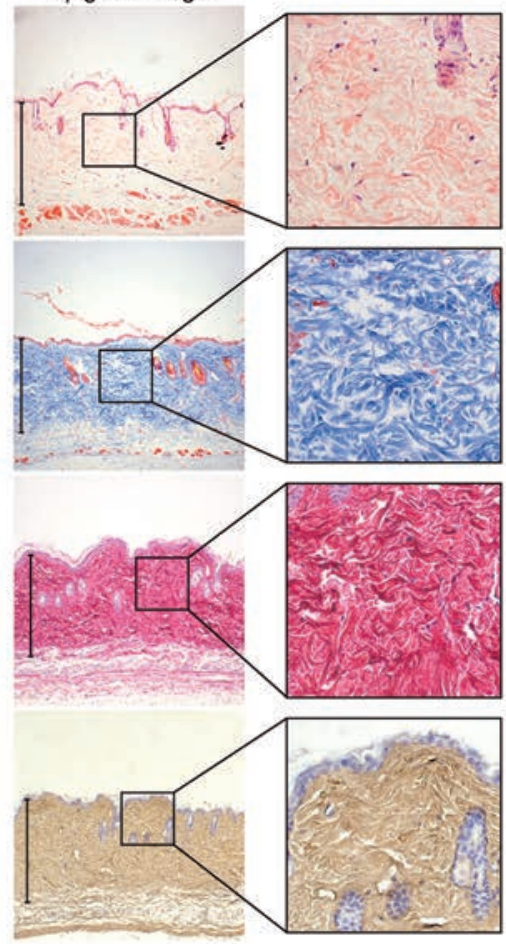

E

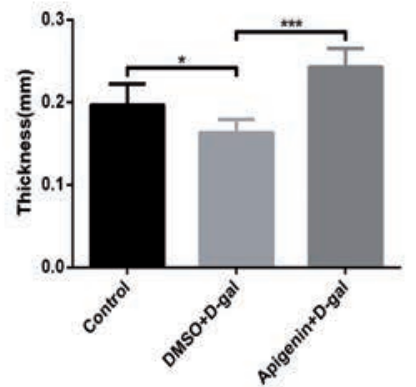

F
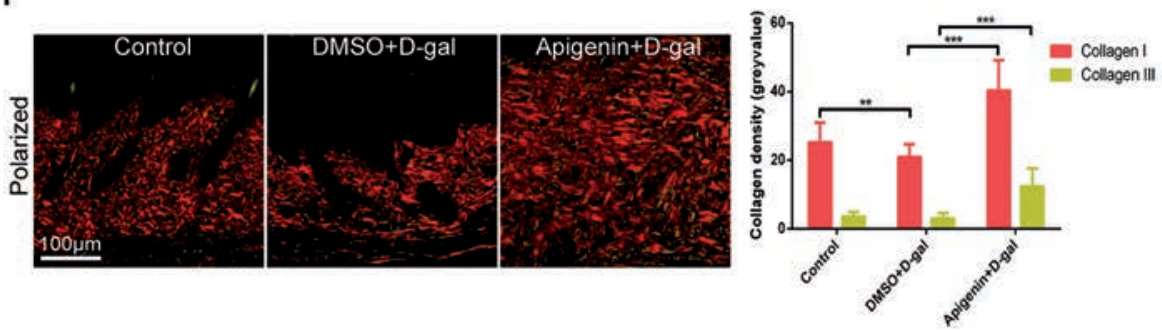

G

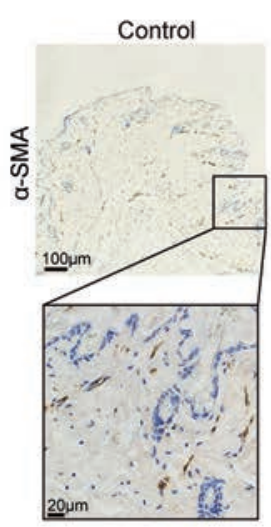

DMSO+D-gal
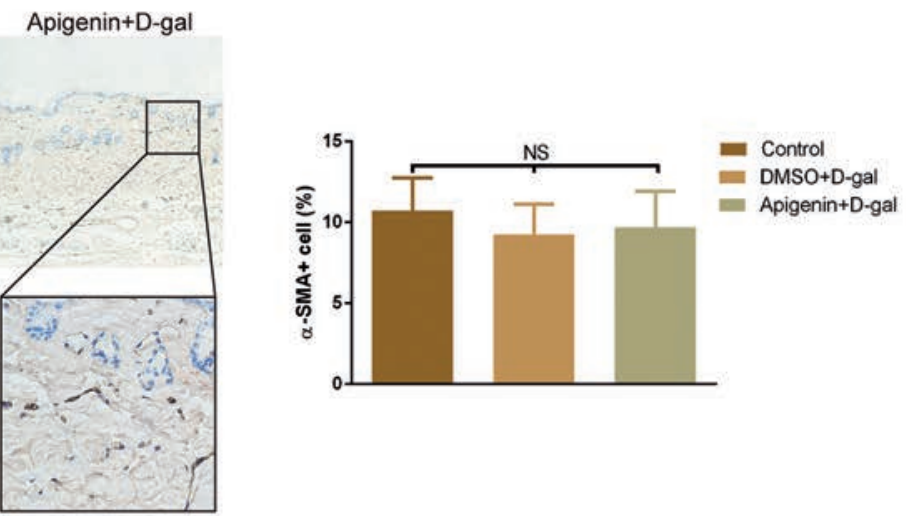

Figure 4. Apigenin increased dermal thickness and collagen density in the D-galactose-induced skin aging mouse model. A-D) H\&E, Masson's trichrome, Picrosirius red and immunohistochemistry stained dermis of control mice and D-gal-treated mice respectively received apigenin and DMSO; scale bars: $100 \mu \mathrm{m}$; zoom scale bars: $20 \mu \mathrm{m}$. E) Quantification of dermal thickness. F) Picrosirius red staining under polarized light and quantification of type I and type III collagen density; collagen type I is shown as red fibers and collagen type III is shown as green fibers; scale bars: $100 \mu \mathrm{m}$. G) Immunohistochemistry staining of $\alpha$-SMA of control mice and D-galtreated mice respectively received apigenin and DMSO; scale bars: $100 \mu \mathrm{m}$; zoom scale bars: $20 \mu \mathrm{m}$. Data are presented as mean \pm SD, $\mathrm{n}=6 / 6 / 6 ;{ }^{*} \mathrm{P}<0.05 ;{ }^{* *} \mathrm{P}<0.01 ;{ }^{* * *} \mathrm{P}<0.001$. 
ty in fibroblasts. We believed that the attenuating effect of high concentration of apigenin on phenotypic transitions in the analyzed cell populations were not independent of its cytotoxic activity. We detected the markers related to extracellular matrix degradation and found that apigenin had no effect on the balance of MMPs/TIMPs.

We further observed that apigenin directly activated smad2/3-dependent signaling pathway. This is not surprising since this flavonoid displays considerable muti-effect. It targets a number of secondary messengers, including those potentially involved in TGF- $\beta 1$ signaling pathway, such as NF-kB, ${ }^{34}$ MAPK/ERK, ${ }^{35}$ FAK, ${ }^{36,37}$ PKC $^{38}$ and PI3K-Akt ${ }^{39}$ in a cell contextdependent manner. We observed that apigenin markedly increased the expression of phosphorylated smad2 and smad3 protein, while total smad2, smad3 and smad4 protein all remained unaltered. So, a more meticulous network may connect TGF- $\beta 1$ signaling pathway and the abovementioned secondary messengers.

A previous study showed the accelerated aging effect of D-gal injection on mouse skin, as well as changes in dermal thickness and collagen content. ${ }^{15}$ In order to confirm the effect of apigenin on collagen synthesis in vivo, the D-galactose-induced skin aging mouse model was established. Our data indicated that skin aging mice treated with apigenin showed markedly increasing dermal thickness and collagen expression, compared with DMSO-treated mice. Hou et al. ${ }^{40}$ reported that topical apigenin improved epidermal permeability barrier function by stimulating epidermal differentiation, lipid synthesis and secretion, as well as cutaneous antimicrobial peptide production, and our result showed that dermal injection of apigenin significantly increased dermal thickness and density. So we could conclude that apigenin caused different biological functions with two forms of drug administration by acting on epidermis or derma, which might indicate the importance of choosing suitable administration methods to different skin diseases, even for the same drug.

Our study demonstrates that apigenin could induce both type I and type III collagen synthesis of fibroblasts in vitro and could increase dermal thickness and collagen deposition in the dermis of mice. This compound is a potential target for drug design and development for esthetic and reconstructive purpose.

\section{References}

1. Baumann L, Kaufman J, Saghari S. Collagen fillers. Dermatol Ther 2006;19: 134-40.
2. Fisher GJ, Wang ZQ, Datta SC, Varani J, Kang S, Voorhees JJ. Pathophysiology of premature skin aging induced by ultraviolet light. N Engl J Med 1997;337:1419-28.

3. Liang JA, Pei XR, Zhang ZF, Wang N, Wang JB, Li Y. The Protective Effects of LongTerm Oral Administration of Marine Collagen Hydrolysate from Chum Salmon on Collagen Matrix Homeostasis in the Chronological Aged Skin of SpragueDawley Male Rats. J Food Sci 2010;75: H230-8.

4. Hou H, Li BF, Zhang ZH, Xue CH, Yu GL, Wang JF, et al. Moisture absorption and retention properties, and activity in alleviating skin photodamage of collagen polypeptide from marine fish skin. Food Chem 2012;135:1432-9.

5. Matsuda N, Koyama YI, Hosaka Y, Ueda H, Watanabe T, Araya T, et al. Effects of ingestion of collagen peptide on collagen fibrils and Glycosaminoglycans in the dermis. $\mathrm{J}$ Nutr Sci Vitaminol (Tokyo) 2006;52:211-5.

6. Iannitti T, Morales-Medina JC, Coacci A, Palmieri B. Experimental and Clinical Efficacy of Two Hyaluronic Acid-based Compounds of Different Cross-Linkage and Composition in the Rejuvenation of the Skin. Pharm Res Epub 2014 Jun 25.

7. Sharma H, Kanwal R, Bhaskaran N, Gupta $\mathrm{S}$. Plant flavone apigenin binds to nucleic acid bases and reduces oxidative DNA damage in prostate epithelial cells. PLoS One 2014;9:e91588.

8. Wang J, Liu YT, Xiao L, Zhu L, Wang Q, Yan T. Anti-Inflammatory Effects of Apigenin in Lipopolysaccharide-Induced Inflammatory in Acute Lung Injury by Suppressing COX2 and NF-kB Pathway. Inflammation 2014;37:2085-90.

9. Polier G, Giaisi M, Kohler R, Muller WW, Lutz C, Buss EC, et al. Targeting CDK9 by wogonin and related natural flavones potentiates the anti-cancer efficacy of the Bcl-2 family inhibitor ABT-263. Int J Cancer 2015;136:688-98.

10. Taupin P. Apigenin and related compounds stimulate adult neurogenesis. Mars, Inc., the Salk Institute for Biological Studies: W02008147483. Expert Opin Ther Pat 2009;19:523-7.

11. Lodhi S, Singhai AK. Wound healing effect of flavonoid rich fraction and luteolin isolated from Martynia annua Linn. on streptozotocin induced diabetic rats. Asian Pac J Trop Med 2013;6:253-9.

12. Singer AJ, Clark RA. Cutaneous wound healing. N Engl J Med 1999;341:738-46.

13. Yano F, Hojo H, Ohba S, Fukai A, Hosaka Y, Ikeda T, et al. A novel disease-modifying osteoarthritis drug candidate targeting Runx1. Ann Rheum Dis 2013;72:748-53.

14. Jin H, Wang $X$, Ying J, Wong AH, Cui Y,
Srivastava G, et al. Epigenetic silencing of a Ca(2+)-regulated Ras GTPase-activating protein RASAL defines a new mechanism of Ras activation in human cancers. Proc Natl Acad Sci U S A 2007;104:12353-8.

15. Zhang S, Dong Z, Peng Z, Lu F. Anti-aging effect of adipose-derived stem cells in a mouse model of skin aging induced by Dgalactose. PLoS One 2014;9:e97573.

16. Prausnitz MR. Microneedles for transdermal drug delivery. Adv Drug Deliv Rev 2004;56:581-7.

17. Wang J, Dodd C, Shankowsky HA, Scott PG, Tredget EE, Wound Healing Research G. Deep dermal fibroblasts contribute to hypertrophic scarring. Lab Invest 2008; 88:1278-90.

18. Verrecchia F, Chu ML, Mauviel A. Identification of novel TGF-beta/Smad gene targets in dermal fibroblasts using a combined cDNA microarray/promoter transactivation approach. J Biol Chem 2001;276:17058-62.

19. Yoon JH, Kim J, Lee H, Kim SY, Jang HH, Ryu SH, et al. Laminin peptide YIGSR induces collagen synthesis in Hs27 human dermal fibroblasts. Biochem Biophys Res Commun 2012;428:416-21.

20. Fenske NA, Lober CW. Structural and functional changes of normal aging skin. J Am Acad Dermatol 1986;15:571-85.

21. Lavker RM. Structural alterations in exposed and unexposed aged skin. J Invest Dermatol 1979;73:59-66.

22. Klein AW, Elson ML. The history of substances for soft tissue augmentation. Dermatol Surg 2000;26:1096-105.

23. Kwok HH, Yue PYK, Mak NK, Wong RNS. Ginsenoside Rb-1 induces type I collagen expression through peroxisome proliferator-activated receptor-delta. Biochem Pharmacol 2012;84:532-9.

24. Winterfield L, Cather J, Cather J, Menter A. Changing paradigms in dermatology: Nuclear hormone receptors Clin Dermatol. 2003;21:447-54.

25. Lee J, Jung E, Yu H, Kim Y, Ha J, Kim YS, et al. Mechanisms of carvacrol-induced expression of type I collagen gene. J Dermatol Sci 2008;52:160-9.

26. Choi MS, Yoo MS, Son DJ, Jung HY, Lee $\mathrm{SH}$, Jung JK, et al. Increase of collagen synthesis by obovatol through stimulation of the TGF-beta signaling and inhibition of matrix metalloproteinase in UVB-irradiated human fibroblast. J Dermatol Sci 2007;46:127-37.

27. Wang J, Zhou J, Zhang N, Zhang X, Li Q. A heterocyclic molecule kartogenin induces collagen synthesis of human dermal fibroblasts by activating the smad4/smad5 pathway. Biochem Biophys Res Commun 2014;450:568-74. 
28. Shukla S, Gupta S. Apigenin: a promising molecule for cancer prevention. Pharm Res 2010;27:962-78.

29. Das S, Das J, Paul A, Samadder A, KhudaBukhsh AR. Apigenin, a bioactive flavonoid from Lycopodium clavatum, stimulates nucleotide excision repair genes to protect skin keratinocytes from ultraviolet B-induced reactive oxygen species and DNA damage. J Acupunct Meridian Stud 2013;6:252-62.

30. Byun S, Park J, Lee E, Lim S, Yu JG, Lee SJ, et al. Src kinase is a direct target of apigenin against UVB-induced skin inflammation. Carcinogenesis 2013;34:397-405.

31. Yano S, Umeda D, Yamashita S, Yamada K, Tachibana H. Dietary apigenin attenuates the development of atopic dermatitis-like skin lesions in NC/Nga mice. J Nutr Biochem 2009;20:876-81.

32. Abu-Yousif A0, Smith KA, Getsios S, Green KJ, Van Dross RT, Pelling JC. Enhancement of UVB-induced apoptosis by apigenin in human keratinocytes and organotypic keratinocyte cultures. Cancer Res 2008;68:3057-65.

33. Ricupero DA, Poliks CF, Rishikof DC, Kuang PP, Goldstein RH. Apigenin decreases expression of the myofibroblast phenotype. FEBS Lett 2001;506:15-21.

34. Kang OH, Lee JH, Kwon DY. Apigenin inhibits release of inflammatory mediators by blocking the NF-kappaB activation pathways in the HMC-1 cells. Immunopharmacol Immunotoxicol 2011; 33:473-9.

35. Hwang YP, Oh KN, Yun HJ, Jeong HG. The flavonoids apigenin and luteolin suppress ultraviolet A-induced matrix metalloproteinase-1 expression via MAPKs and AP-1dependent signaling in HaCaT cells. $\mathrm{J}$ Dermatol Sci 2011;61:23-31.

36. Franzen CA, Amargo E, Todorovic V, Desai BV, Huda S, Mirzoeva S, et al. The Chemopreventive Bioflavonoid Apigenin Inhibits Prostate Cancer Cell Motility through the Focal Adhesion Kinase/Src Signaling Mechanism. Cancer Prev Res
(Phila) 2009;2:830-41.

37. Hu XW, Meng D, Fang J. Apigenin inhibited migration and invasion of human ovarian cancer A2780 cells through focal adhesion kinase. Carcinogenesis 2008;29:2369-76.

38. Balasubramanian S, Zhu L, Eckert RL. Apigenin inhibition of involucrin gene expression is associated with a specific reduction in phosphorylation of protein kinase C delta Tyr(311). J Biol Chem 2006;281:36162-72.

39. Shukla S, Gupta S. Apigenin-induced cell cycle arrest is mediated by modulation of MAPK, PI3K-Akt, and loss of cyclin D1 associated retinoblastoma dephosphorylation in human prostate cancer cells. Cell Cycle 2007;6:1102-14.

40. Hou M, Sun R, Hupe M, Kim PL, Park K, Crumrine D, et al. Topical apigenin improves epidermal permeability barrier homoeostasis in normal murine skin by divergent mechanisms. Exp Dermatol 2013;22:210-5. 